\section{Case Reports in Oncology}

\title{
A Novel PRKAR1A Mutation Identified in a Patient with Isolated Primary Pigmented Nodular Adrenocortical Disease
}

\author{
Sira Korpaisarn ${ }^{a}$ Objoon Trachoo ${ }^{a, c} \quad$ Bhakbhoom Panthan ${ }^{b, c}$ \\ Rangsima Aroonroch ${ }^{b}$ Ronnarat Suvikapakornkul ${ }^{d}$ \\ Chutintorn Sriphrapradang ${ }^{a}$
}

${ }^{a}$ Department of Medicine, Faculty of Medicine Ramathibodi Hospital, Mahidol University, Bangkok, Thailand; ${ }^{b}$ Department of Pathology, Faculty of Medicine Ramathibodi Hospital, Mahidol University, Bangkok, Thailand; ' ${ }^{C}$ Center for Medical Genomics, Faculty of Medicine Ramathibodi Hospital, Mahidol University, Bangkok, Thailand; ${ }^{d}$ Department of Surgery, Faculty of Medicine Ramathibodi Hospital, Mahidol University, Bangkok, Thailand

\section{Keywords}

Adrenal adenoma $\cdot$ Cushing syndrome $\cdot$ Mutation $\cdot$ Penetrance $\cdot$ Splice site

\begin{abstract}
Primary pigmented nodular adrenocortical disease (PPNAD) is a rare cause of Cushing syndrome, especially the isolated form without Carney complex, associated with germline mutations in PRKAR1A, the protein kinase A regulatory subunit type 1 alpha gene. We report a 31year-old female who presented with secondary amenorrhea, cushingoid appearance, and hypertension without Carney complex. Biochemical laboratory examinations confirmed the $\mathrm{ACTH}$-independent adrenal Cushing syndrome with negative Liddle test. A small right adrenal adenoma of $0.8 \mathrm{~cm}$ was shown on computed tomography while magnetic resonance imaging revealed nodularity of both adrenal glands. The histological report confirmed PPNAD using laparoscopic right adrenalectomy, and subsequent left adrenalectomy was performed 6 months later. She had inherited heterozygosity of a novel germline mutation of the PRKAR1A gene (g.114213T $>$ G or c.709-5T $>$ G). This splice site mutation results in exon 8
\end{abstract}

KARGER
Chutintorn Sriphrapradang, MD

Department of Medicine, Faculty of Medicine Ramathibodi Hospita

Mahidol University, 270 Rama 6 Road

Bangkok 10400 (Thailand)

E-Mail chutins@gmail.com 


\section{Case Reports in Oncology}

skipping. Her father carrying the same mutation had no clinical features of either PPNAD or Carney complex. This novel PRKAR1A gene mutation, c.709-5T>G, is reported here for the first time manifesting as an incomplete clinical expression of the isolated form of PPNAD and being inherited with low penetrance unlike other inherited mutations of the Carney complex which have a penetrance of almost $100 \%$.

\section{Introduction}

Primary pigmented nodular adrenocortical disease (PPNAD) is a very rare cause of ACTH-independent Cushing syndrome (CS). Bilateral adrenal glands are involved containing numerous small pigmented nodules. PPNAD usually presents in the context of Carney complex (CNC), a multiple neoplasia syndrome featuring a complex of myxoma, spotty pigmentation, and endocrine overactivity. In addition to PPNAD, which is the most common endocrine disorder in $\mathrm{CNC}$, other endocrine tumors include pituitary adenomas, thyroid adenomas or carcinomas, ovarian cysts, and large-cell calcifying Sertoli cell tumors [1]. Ten to twenty percent of PPNAD patients show no other neoplasia or familial history, which is known as isolated PPNAD (iPPNAD) [2]. PPNAD is frequently diagnosed in children and adolescents with a peak in the second to third decade of life [1]. PPNAD often expresses as atypical CS; however, classical CS and cyclical CS can be a clinical manifestation [3, 4]. Unlike classical CS, atypical CS shows asthenia with osteoporosis, short stature, and muscle and skin wasting. Cyclical CS is a condition where hypercortisolism occurs episodically only for weeks to months alternating with the normal state. This subtle expression may underlie PPNAD to be underrecognized.

CNC is an autosomal dominant hereditary disease with a penetrance of almost $100 \%$. Thus far, two genetic loci have been linked to this syndrome, 2 p16 and 17q22-24 [5, 6]. In the latter locus, heterozygous mutations of PRKAR1A gene, a tumor suppressor gene, were identified in $65-80 \%$ of CNC patients [7]. PRKAR1A encodes the key molecule in multiple cAMP-mediated endocrine signaling pathways, the type 1 alpha regulatory subunit of protein kinase A. Overactivity of protein kinase $\mathrm{A}$, as a consequence of inactivating mutation of PRKAR1A, results in tumorigenesis in affected tissues. Several germline mutations in PRKAR1A have been linked with iPPNAD. One of those was identified as a "hot spot," c.709 (-7-2)del6 mutation, resulting in abnormal splicing and exon 8 skipping as a final consequence [8]. However, it is unclear why the disease is limited only to adrenal glands.

We report a 31-year-old woman who presented with secondary amenorrhea, hypertension, and cushingoid appearance due to iPPNAD. She inherited heterozygosity of a novel PRKAR1A mutation, c.709-5T>G. This novel mutation is located at the same locus as the hot spot mutation and likewise leads to alteration of splicing and exon 8 skipping.

\section{Clinical Report}

A 31-year-old female presented with secondary amenorrhea of 5 months' duration. Her weight had increased by $4 \mathrm{~kg}$ (6.3\% from her normal weight) in 4 months and she experienced acne on her face. Her skin became more fragile and easily bruised even with minor traumas. She had been on antihypertensive medication for 3 years including atenolol 100 $\mathrm{mg} /$ day and losartan $100 \mathrm{mg}$ /day for inadequately controlled blood pressure. She denied drinking alcohol or using an exogenous steroid. Her family history was unremarkable for CS 


\section{Case Reports in Oncology}

or tumor disease. On examination, she had a significant cushingoid appearance with a round face, thin skin, supraclavicular fat pad, and proximal muscle weakness. There were purplish striae on her lower abdomen and numerous acne lesions on her face. She weighed $56 \mathrm{~kg}$ and her body mass index was 22.7. Her blood pressure was slightly elevated at 145/95 mm Hg. Cardiac examination was unremarkable along with the chest, breast, and abdominal examination.

Laboratory investigations revealed leukocytosis predominant with neutrophils and hypokalemia of $3.36 \mathrm{mmol} / \mathrm{L}$ (normal range: 3.50-5.10 mmol/L). Her fasting blood glucose, creatinine, and liver function test were all normal. The 24-h urinary free cortisol was high with a level of $335.04 \mu \mathrm{g} /$ day (normal range: $24-143 \mu \mathrm{g} /$ day). A 2-day low-dose dexamethasone suppression test ( $0.5 \mathrm{mg}$ of dexamethasone every $6 \mathrm{~h}$ ) was unable to suppress an adrenal function with the morning serum cortisol level of $25.5 \mu \mathrm{g} / \mathrm{dL}$. The serum ACTH level was undetectable with the level being less than $10 \mathrm{pg} / \mathrm{mL}$. Accordingly, ACTH-independent CS was diagnosed.

Adrenal computed tomography showed an enlarged lateral limb of the right adrenal gland with a nodular lesion at the superior aspect of $0.8 \mathrm{~cm}$ in size. The lesion was 34 Hounsfield units in the unenhanced study with a $51 \%$ absolute washout. The left adrenal gland appeared to be normal (Fig. 1). The right adrenal adenoma was suspected but a hepatic nodule was also a possibility. Further magnetic resonance imaging of adrenal glands was suggested. Magnetic resonance imaging revealed mild nodularity of both adrenal glands and the dominant discrete nodule at the superior aspect of the right adrenal gland with $0.5 \mathrm{~cm}$ in size. Conclusively, the findings suggested PPNAD. Since the diagnosis was controversial between PPNAD and adrenal adenoma, the Liddle test and adrenal venous sampling were done. The Liddle test was unable to show a paradoxical rising in urine free cortisol, which typically associates with PPNAD. Unfortunately, the right adrenal vein was unable to be adequately accessed during adrenal venous sampling yielding invalid results. Both additional investigations did not reveal a clear diagnosis of either PPNAD or adrenal adenoma.

There was no evidence of CNC, with which most PPNADs are associated. No skin hyperpigmentation was observed and an echocardiogram was also unremarkable. There was no thyroid nodule found. Up to this point, right adrenalectomy was planned. Contralateral adrenalectomy was also discussed but the decision to perform it depended upon the pathological report. Ketoconazole, $400 \mathrm{mg} /$ day, was started preoperatively. The preoperative evaluation disclosed pulmonary tuberculosis, and hence the patient completed a 6-month course of antituberculosis medication.

The pathological report showed hyperpigmentation in the right adrenal gland, which weighed $5.4 \mathrm{~g}$ and was $5 \times 3 \times 1.5 \mathrm{~cm}$ in size (Fig. 2). This was consistent with PPNAD. After surgery, her menstruation became regular. Her round face, easily bruised skin, and proximal myopathy all improved significantly. The dose of antihypertensive drugs was reduced by $50 \%$. However, a repeated overnight 1-mg dexamethasone suppression test was unable to suppress morning cortisol level, $7.5 \mu \mathrm{g} / \mathrm{dL}$. Left adrenalectomy was done 6 months later and confirmed the PPNAD. The left adrenal gland weighed $4.5 \mathrm{~g}$ and was $5 \times 3 \times 1.5 \mathrm{~cm}$ in size. An external surface showed a yellow-brown nodular appearance. Cut sections revealed multiple cortical nodular solid black nodules of $0.2-0.4 \mathrm{~cm}$ in size (Fig. 2). Consequently, all her symptoms gradually improved. Prednisolone and fludrocortisone were started to replace her adrenal function. 
 Oncology}

\section{Genetic Analyses of PRKAR1A}

Informed consent was obtained from the patient and her relatives. This study was approved by the Institutional Review Board. Genomic DNA of the patient and her relatives was extracted from peripheral blood leukocytes. Twelve exons and flanking intronic sequences of the PRKAR1A gene were analyzed. DNA sequencing of PRKAR1A demonstrated the heterozygosity of a novel PRKAR1A germline mutation, g.114213T $>\mathrm{G}$ or c.709-5T $>\mathrm{G}$, in the patient and her father. Her mother and sister carry the normal PRKAR1A sequence. This mutation is a substitution of thiamine by guanine at g.114213, proximal to exon 8 by 5 bases.

Exon 8 skipping is a predicted sequela of this mutation; therefore, further RT-PCR was performed using primers flanking exon 8 used by Groussin et al. [8]. RT-PCR of exon 5-10 in the patient showed two products (Fig. 3). One product was also found in normal controls. The other, which was much less intense, was only found in the patient and likely secondary from this novel mutation resulted exon 8 skipping. In order to prove that exon 8 skipping is an underlying sequela of this mutation, additional RT-PCR was performed using specific primers: sense primer $5^{\prime}$-AATGGCCGCTTTAGCCAAAGCC-3', matching with exon 5, and antisense primer $5^{\prime}$-ACGTTCCCACTTGTCCAGAGACTCAT- $3^{\prime}$ (the bold sequence at $3^{\prime}$ is specific for exon 9 while the rest match with exon 7). The result is shown in Figure 3, yielding the specific product of exon 5-7 in the patient, compatible with exon 8 skipping.

\section{Discussion}

PPNAD usually presents along with CNC while $12 \%$ of the cases manifest as a sole presentation, known as iPPNAD [9]. Like CNC, several inactivating heterozygous germline mutations of PRKAR1A were identified associating with iPPNAD and most of them are de novo mutations. One of those was considered as a hot spot, c.709(-7-2)del6, leading to 6-bp polypyrimidine tract (TTTTTA) deletion in intron 7 . This mutation alters the polypyrimidine rich tract adjacent to a $3^{\prime}$ splice site of the intron while preserving the splice acceptor site. The shortened polypyrimidine rich tract affects the $3^{\prime}$ splice site recognition resulting in abnormal splicing and exon 8 skipping as a final consequence [2]. Groussin et al. [8] reported this hot spot mutation in 12 iPPNAD patients from 12 unrelated families. This hot spot is one of few mutations showing a clear genotype-phenotype correlation of PRKAR1A specific to iPPNAD. Recently, it has been discovered that iPPNAD relates to another 2 genes, which code for phosphodiesterases type 11A (PDE11A) and type 8B (PDE8B) [10,11]. Inactivating mutations in both loci also involve hyperfunction of the cAMP signaling pathway.

The present clinical vignette carries the novel heterozygous mutation, c.709-5T>G, which is located exactly at the same locus as the previously mentioned hot spot mutation. RT-PCR using primers specific to exon 8 skipping generated specific products in the patient. The sense primer matches exon 5 while the antisense primer attaches precisely to exon 7-9, skipping exon 8. Normal control rarely produced a specific product since the antisense primer did not pair with the normal PRKAR1A mRNA sequence, containing exon 8 . This proves that this novel mutation leads to exon 8 skipping as a final consequence, which is similar to the hot spot mutation, c.709(-7-2)del6, mentioned above.

CNC was described as of autosomal dominant heredity with a penetrance of almost $100 \%$ [1]. However, c.709(-7-2)del6 mutation leading to iPPNAD has a low penetrance [8], and this reported novel mutation may carry the same characteristic. In this present clinical vignette, the patient's father carries the heterozygosity of this novel mutation but, due to an 
incomplete penetrance, there is no phenotypic manifestation. The tissue-specific microenvironmental factors were described as a potential tumorigenic modified factor of iPPNAD phenotype in different individuals, for example, female patients after puberty. While the PRKAR1A expression has no gender difference, there is a gender preponderance of female over male only after puberty in PPNAD patients with PRKAR1A mutation [9]. This explains why our patient manifested with CS in her 30s but her father, who carries the same mutation but without tumorigenic modified factor, remains asymptomatic.

Total bilateral adrenalectomy is a standard treatment for bilateral adrenal CS, both ACTH-independent macronodular adrenal hyperplasia and PPNAD. Recent studies showed that unilateral adrenalectomy in ACTH-independent macronodular adrenal hyperplasia and PPNAD may result in long-term remission in CS [12, 13]. Xu et al. [13] reported that 12 out of 13 PPNAD patients had clinical and laboratory remission of CS after unilateral adrenalectomy at a median follow-up of 47 months. The present report also showed a significant clinical improvement after unilateral adrenalectomy. However, total adrenalectomy was finally preformed due to a failure to suppress the cortisol level with overnight $1 \mathrm{mg}$ dexamethasone for 6 months after unilateral adrenalectomy.

In conclusion, we report the novel PRKAR1A mutation, c.709-5T $>\mathrm{G}$, causing the restricted phenotype of iPPNAD. This splice site mutation leads to abnormal splicing and eventually exon 8 skipping. Though most of PRKAR1A mutations are above a penetrance of $95 \%$ by the age of 50 years, this novel mutation is likely one of very few mutations where incomplete penetrance is observed. Due to a subtle presentation of iPPNAD, the genetic analysis might be helpful to facilitate a diagnosis and surveillance in families which inherited this mutation.

\section{Statement of Ethics}

Informed consent was obtained from the patient and her relatives. This study was approved by the Institutional Review Board.

\section{Disclosure Statement}

The authors declare that they have no conflicts of interest.

\section{Funding Sources}

This study was supported by grants of the Rare Genetic Disorder Funds, Department of Medicine, Faculty of Medicine Ramathibodi Hospital, Mahidol University.

\section{References}

1 Stratakis CA, Kirschner LS, Carney JA: Clinical and molecular features of the Carney complex: diagnostic criteria and recommendations for patient evaluation. J Clin Endocrinol Metab 2001;86:4041-4046.

-2 Cazabat L, Ragazzon B, Groussin L, Bertherat J: PRKAR1A mutations in primary pigmented nodular adrenocortical disease. Pituitary 2006;9:211-219.

3 Mellinger RC, Smith RW Jr: Studies of the adrenal hyperfunction in 2 patients with atypical Cushing's syndrome. J Clin Endocrinol Metab 1956;16:350-366. 
-4 Horvath A, Stratakis C: Primary pigmented nodular adrenocortical disease and Cushing's syndrome. Arq Bras Endocrinol Metabol 2007;51:1238-1244.

-5 Stratakis CA, Carney JA, Lin JP, Papanicolaou DA, Karl M, Kastner DL, Pras E, Chrousos GP: Carney complex, a familial multiple neoplasia and lentiginosis syndrome. Analysis of 11 kindreds and linkage to the short arm of chromosome 2. J Clin Invest 1996;97:699-705.

-6 Casey M, Mah C, Merliss AD, Kirschner LS, Taymans SE, Denio AE, Korf B, Irvine AD, Hughes A, Carney JA, Stratakis CA, Basson CT: Identification of a novel genetic locus for familial cardiac myxomas and Carney complex. Circulation 1998;98:2560-2566.

-7 Veugelers M, Wilkes D, Burton K, McDermott DA, Song Y, Goldstein MM, La Perle K, Vaughan CJ, O’Hagan A, Bennett KR, Meyer BJ, Legius E, Karttunen M, Norio R, Kaariainen H, Lavyne M, Neau JP, Richter G, Kirali K, Farnsworth A, Stapleton K, Morelli P, Takanashi Y, Bamforth JS, Eitelberger F, Noszian I, Manfroi W, Powers J, Mochizuki Y, Imai T, Ko GT, Driscoll DA, Goldmuntz E, Edelberg JM, Collins A, Eccles D, Irvine AD, McKnight GS, Basson CT: Comparative PRKAR1A genotype-phenotype analyses in humans with Carney complex and prkar1a haploinsufficient mice. Proc Natl Acad Sci USA 2004;101:14222-14227.

-8 Groussin L, Horvath A, Jullian E, Boikos S, Rene-Corail F, Lefebvre H, Cephise-Velayoudom FL, Vantyghem MC, Chanson P, Conte-Devolx B, Lucas M, Gentil A, Malchoff CD, Tissier F, Carney JA, Bertagna X, Stratakis CA, Bertherat J: A PRKAR1A mutation associated with primary pigmented nodular adrenocortical disease in 12 kindreds. J Clin Endocrinol Metab 2006;91:1943-1949.

-9 Bertherat J, Horvath A, Groussin L, Grabar S, Boikos S, Cazabat L, Libe R, Rene-Corail F, Stergiopoulos S, Bourdeau I, Bei T, Clauser E, Calender A, Kirschner LS, Bertagna X, Carney JA, Stratakis CA: Mutations in regulatory subunit type $1 \mathrm{~A}$ of cyclic adenosine $5^{\prime}$-monophosphate-dependent protein kinase (PRKAR1A): phenotype analysis in 353 patients and 80 different genotypes. J Clin Endocrinol Metab 2009;94:2085-2091.

-10 Horvath A, Boikos S, Giatzakis C, Robinson-White A, Groussin L, Griffin KJ, Stein E, Levine E, Delimpasi G, Hsiao HP, Keil M, Heyerdahl S, Matyakhina L, Libe R, Fratticci A, Kirschner LS, Cramer K, Gaillard RC, Bertagna X, Carney JA, Bertherat J, Bossis I, Stratakis CA: A genome-wide scan identifies mutations in the gene encoding phosphodiesterase 11A4 (PDE11A) in individuals with adrenocortical hyperplasia. Nat Genet 2006;38:794-800.

11 Horvath A, Mericq V, Stratakis CA: Mutation in PDE8B, a cyclic AMP-specific phosphodiesterase in adrenal hyperplasia. N Engl J Med 2008;358:750-752.

$\checkmark 12$ Iacobone M, Albiger N, Scaroni C, Mantero F, Fassina A, Viel G, Frego M, Favia G: The role of unilateral adrenalectomy in ACTH-independent macronodular adrenal hyperplasia (AIMAH). World J Surg 2008;32:882-889.

13 Xu Y, Rui W, Qi Y, Zhang C, Zhao J, Wang X, Wu Y, Zhu Q, Shen Z, Ning G, Zhu Y: The role of unilateral adrenalectomy in corticotropin-independent bilateral adrenocortical hyperplasias. World J Surg 2013;37:1626-1632. 


\section{Case Reports in Oncology}
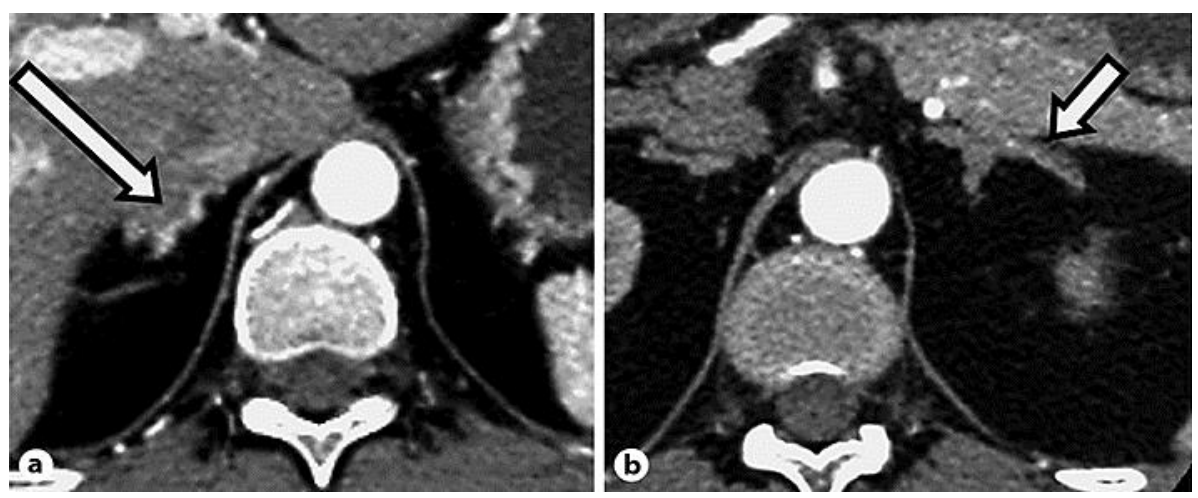

Fig. 1. Computed tomography of adrenal glands. a The white arrow shows the enlarged lateral limb of the right adrenal gland with the nodular lesion at the superior aspect of $0.8 \mathrm{~cm}$ in size. The lesion was 34 Hounsfield units on the noncontrast study with 51\% absolute washout. b The white arrow shows the left adrenal gland which appears unremarkable.

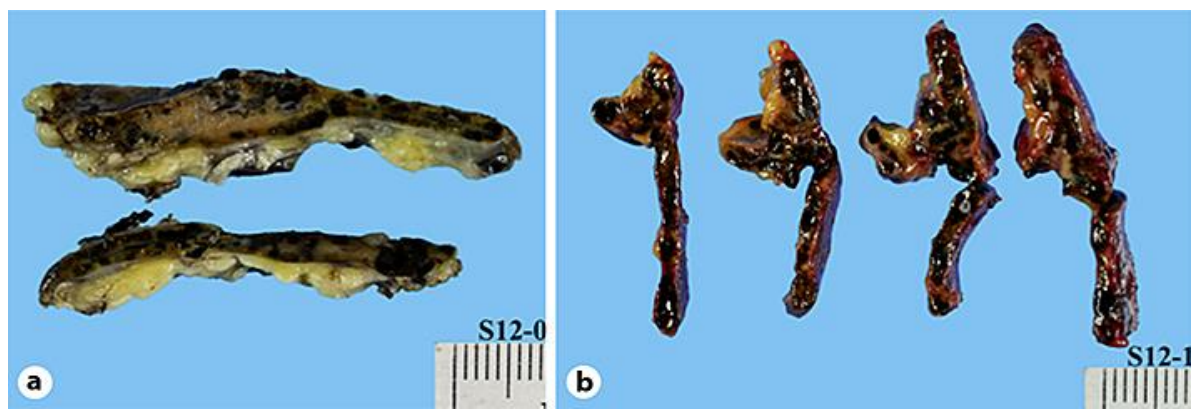

Fig. 2. a Right adrenal gland: an external surface showed a smooth shiny yellow appearance. b Left adrenal gland: an external surface showed a yellow-brown nodular appearance. Cut sections revealed multiple cortical nodular solid black nodules of $0.2-0.4 \mathrm{~cm}$ in size. 


\section{Case Reports in Oncology}

a

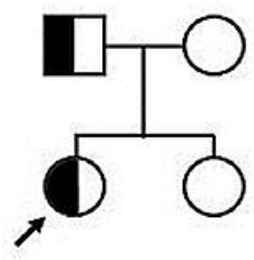

MW Patient Control

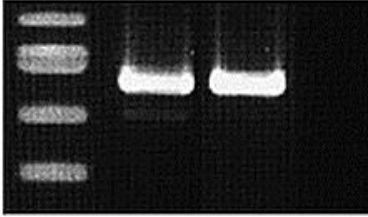

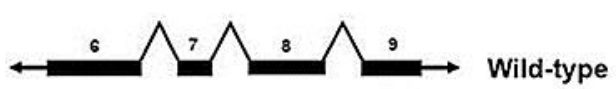

b

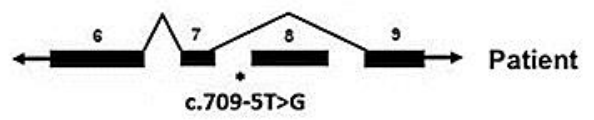

\section{$\leftarrow$ Wild-type}

Exon 8

skipping

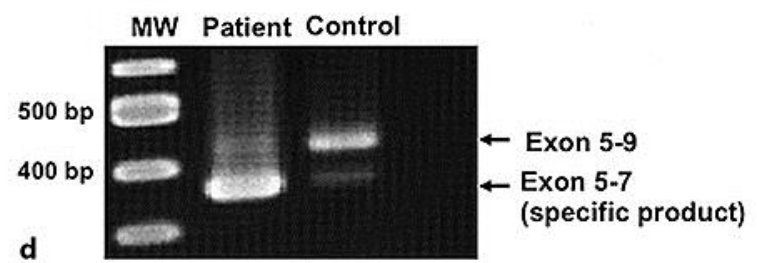

Fig. 3. a Pedigree of the family of the patient. The arrow indicates the proband who is the only one manifesting as iPPNAD. $\mathbf{b}$ Schematic of coding exons and splicing patterns of the PRKAR1A gene in the wild type and the patient. Exon 8 skipping occurs in the patient. The asterisk indicates the locus of the novel mutation. c RT-PCR using the primers flanking exon 8. RT-PCR of exon 5-10 in the patient showed two products. The upper band is the wild type (463 bp) which is also found in normal controls. The lower band is much less intense as a result of abnormal splicing resulting in exon 8 skipping. $\mathbf{d}$ RT-PCR using the primers specific to exon 8 skipping mRNA. Sense primer matches with exon 5 and antisense primer straddles on exon 7 and exon 9. The lower band is the specific product of exon 5-7 (360 bp), which results from these particular primers and was found in our patient. The upper band is the nonspecific product of exon 5-9 (421 bp) which was found in normal controls. Since the specific product was revealed in this patient, this novel mutation likely causes exon 8 skipping. 